\title{
A Facile Synthesis of Graphene-WO $\mathrm{O}_{3}$ Nanowire Clusters with High Photocatalytic Activity for $\mathbf{O}_{2}$ Evolution
}

\author{
M.-J. Zhou, N. Zhang, and Z. H. Hou \\ School of Chemistry and Chemical Engineering, Hunan Institute of Science and Technology, Yueyang 414000, China \\ Correspondence should be addressed to Z.H. Hou; zhqh96@163.com
}

Received 1 April 2014; Revised 24 June 2014; Accepted 8 July 2014; Published 24 July 2014

Academic Editor: Gianluca Li Puma

Copyright (C) 2014 M.-J. Zhou et al. This is an open access article distributed under the Creative Commons Attribution License, which permits unrestricted use, distribution, and reproduction in any medium, provided the original work is properly cited.

In the present work, graphene- $\mathrm{WO}_{3}$ nanowire clusters were synthesized via a facile hydrothermal method. The obtained graphene- $\mathrm{WO}_{3}$ nanowire clusters were characterized by X-ray diffraction (XRD), transmission electron microscopy (TEM), Xray photoelectron spectroscopy (XPS), Fourier transform infrared spectroscopy (FT-IR), Raman spectroscopy, and ultravioletvisible diffuse reflectance spectroscopy (DRS) techniques. The photocatalytic oxygen $\left(\mathrm{O}_{2}\right)$ evolution properties of the as-synthesized samples were investigated by measuring the amount of evolved $\mathrm{O}_{2}$ from water splitting. The graphene- $\mathrm{WO}_{3}$ nanowire clusters exhibited enhanced performance compared to pure $\mathrm{WO}_{3}$ nanowire clusters for $\mathrm{O}_{2}$ evolution. The amount of evolved $\mathrm{O}_{2}$ from water splitting after $8 \mathrm{~h}$ for the graphene- $\mathrm{WO}_{3}$ nanowire clusters is $c a .0 .345 \mathrm{mmol} / \mathrm{L}$, which is more than 1.9 times as much as that of the pure $\mathrm{WO}_{3}$ nanowire clusters $(\mathrm{ca} .0 .175 \mathrm{mmol} / \mathrm{L})$. The high photocatalytic activity of the graphene- $\mathrm{WO}_{3}$ nanowire clusters was attributed to a high charge transfer rate in the presence of graphene.

\section{Introduction}

Since Fujishima and Honda reported the evolution of oxygen and hydrogen from a $\mathrm{TiO}_{2}$ electrode under the irradiation of light in 1972 [1], photocatalysis is a very promising process to photodissociate water utilizing solar energy. Solar photolysis of water is one of the cleanest ways of producing hydrogen and oxygen, which has great potential in solving energy problem. Recently, tungsten oxide $\left(\mathrm{WO}_{3}\right)$ as an important photocatalytic material with a wide band gap ranging from 2.4 to $2.8 \mathrm{eV}$ has attracted considerable interest because it has the potential ability to promote photocatalytic reactions under visible light irradiation [2-4]. The photocatalytic applications of several types of $\mathrm{WO}_{3}$ nanomaterials have been reported, particularly for oxygen $\left(\mathrm{O}_{2}\right)$ evolution in the presence of an electron accepter [5, 6]. However, $\mathrm{WO}_{3}$ nanomaterials are usually not efficient photocatalysts because of the high electron-hole recombination rate [7]. This is one of the biggest obstacles hindering the development of $\mathrm{WO}_{3}$ as a practical photocatalyst [8]. Recently, many attempts have been made to improve the efficiency of electron-hole pair separation in $\mathrm{WO}_{3}$, such as morphology control, doping, and composites [9].
Recently, much effort has been focused on the synthesis of graphene- (GE-) based composites due to their potential application in the photocatalytic field [10-28]. The combination of GE with a well photocatalytic semiconductor is expected to result in a high performance in photocatalytic activity because GE has perfect two-dimensional carbon structure with high thermal conductivity and a large specific surface area [10]. GE is easy to produce from graphene oxide $(\mathrm{GO})$. The presence of oxygen-containing functional group in GO and reduced GO makes it as excellent supporter to anchor photocatalytic semiconductors for the synthesis of GE-based composites. In general, these composites are prepared from the reduction of GO-based materials through a chemical method or heat treatment. However, these methods not only use toxic hydrazine hydrate, but also suffer from some harsh conditions.

To the best of our knowledge, no investigation concerning the nanocomposite which consisted of $\mathrm{WO}_{3}$ nanowire clusters and GE nanosheets for water splitting has been reported. Guo et al. reported that $\mathrm{WO}_{3} @ \mathrm{GE}$ composite showed improved photocatalytic activity to drive the watersplitting reaction to produce oxygen [25]. $\mathrm{Ng}$ et al. incorporated reduced graphene oxide with $\mathrm{WO}_{3}$ nanoparticles, 
achieving 1.6 times improvement in the photocurrent generation [26]. In the present work, $\mathrm{GE}-\mathrm{WO}_{3}$ nanowire clusters were synthesized via a facile hydrothermal method. First, the structures and morphologies of the as-synthesized samples were characterized by X-ray diffraction (XRD), transmission electron microscopy (TEM), X-ray photoelectron spectroscopy (XPS), Fourier transform infrared spectroscopy (FT-IR), Raman spectroscopy, and ultraviolet-visible diffuse reflectance spectroscopy (DRS) techniques. Then, the photocatalytic $\mathrm{O}_{2}$ evolution properties of the samples were investigated by measuring the amount of evolved $\mathrm{O}_{2}$ from water splitting.

\section{Experimental}

2.1. Synthesis of GE-WO $\mathrm{O}_{3}$ Nanowire Clusters. All of the chemical reagents were analytical grade and were used without further purification. GO was synthesized from natural graphite powder based on modified Hummers method [11]. GE-WO nanowire clusters were synthesized via a facile hydrothermal method. Typically, $\mathrm{Na}_{2} \mathrm{WO}_{4} \cdot 2 \mathrm{H}_{2} \mathrm{O}$ and polyethylene glycol (PEG-4000) were directly dissolved in deionized water, and the $\mathrm{pH}$ value of the solution was adjusted to 1.5 adding $\mathrm{HCl} / \mathrm{NaOH}$ solution. Different weight ratios between GO powders and $\mathrm{WO}_{3}$ nanowire clusters (at $1.0 \mathrm{wt} \%, 1.5 \mathrm{wt} \%$, $2.0 \mathrm{wt} \%, 3.0 \mathrm{wt} \%$, and $4.0 \mathrm{wt} \%)$ were dispersed rapidly in ethanol with ultrasonic. Then, the two solutions were mixed and transferred into the Teflon-lined stainless steel autoclave with a capacity of $100 \mathrm{~mL}$. Hydrothermal treatments were carried out at $180^{\circ} \mathrm{C}$ for $30 \mathrm{~h}$. After that, the autoclave was allowed to cool down naturally. Subsequently, the products were collected and washed with deionized water and ethanol several times and dried at $70^{\circ} \mathrm{C}$ for $12 \mathrm{~h}$ in air. Finally, the products were annealed at $400^{\circ} \mathrm{C}$ for $4 \mathrm{~h}$ under nitrogen atmosphere. Pure $\mathrm{WO}_{3}$ nanowire clusters were also obtained through a similar procedure only in the absence of GO.

2.2. Characterization. The XRD patterns obtained on a $\mathrm{D} / \mathrm{max}-\amalg \mathrm{X}$-ray diffractometer using $\mathrm{Cu} \mathrm{K} \alpha$ radiation $(\lambda=$ $1.5418 \AA$ ) at a scan speed of $0.05^{\circ} \mathrm{s}^{-1}$ were used to determine phase structure. The morphologies of the samples were evaluated by transmission electron microscopy (TEM, JEOL JEM-2010 at $200 \mathrm{kV}$ ). Fourier transform infrared (FT-IR) spectroscopy was recorded on a Bruker VECTOR22 FT-IR spectrometer using $\mathrm{KBr}$ pellets. Chemical bonding between the functional groups and carbon atoms was confirmed by X-ray photoelectron spectroscopy (XPS, PHI 5000C ESCA System). Raman spectra were recorded on a microscopic confocal Raman spectrometer (Renishaw 1000 NR). The UVvis spectra were obtained via UV-visible spectrophotometer (Shimadzu UV-2500, Japan).

2.3. Photocatalytic Oxygen Evolution Experiments. Photocatalytic oxidation reactions were carried out in a self-made Lab Solar gas photocatalysis system with external light irradiation. The reaction temperature was kept at ca. $25^{\circ} \mathrm{C}$ using the temperature-controlled cooling water. The light source was a $150 \mathrm{~W}$ xenon lamp with $\lambda=400 \sim 700 \mathrm{~nm}$ and was used

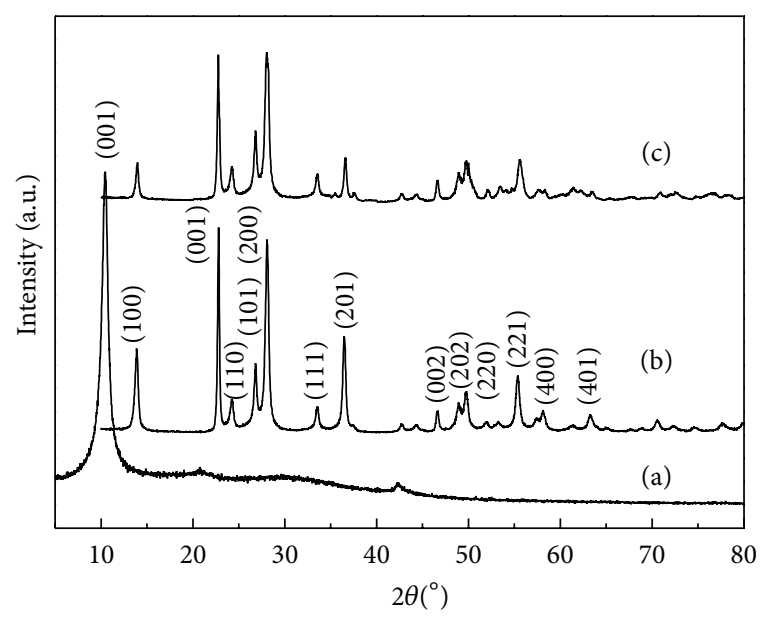

FIGURE 1: XRD patterns of (a) GO, (b) $\mathrm{WO}_{3}$ nanowire clusters, and (c) $\mathrm{GE}-\mathrm{WO}_{3}$ nanowire clusters.

as the simulated sunlight source (CEL-HXUV150, China). Before the photochemical reaction, $500 \mathrm{~mL}$ of deionized water was degassed by boiling it for $0.5 \mathrm{~h}$ and cooling to room temperature and then adding it to the reactor. Then, $2 \mathrm{~g}$ of the obtained photocatalysts and $100 \mathrm{~mL}$ of $0.1 \mathrm{~mol} / \mathrm{L}$ $\mathrm{Fe}_{2}\left(\mathrm{SO}_{4}\right)_{3}$ were added to the reactor under magnetic vigorous stirring to ensure the mixture suspense, using $\mathrm{H}_{2} \mathrm{SO}_{4}$ solution to adjust the $\mathrm{pH}$ of the mixture at 2. The amount of $\mathrm{O}_{2}$ evolved was determined using gas chromatography (TCD: thermal conductivity detector, nitrogen carrier gas).

\section{Results and Discussion}

Figure 1 shows the XRD patterns of the as-synthesized samples. As for the GO, the sharp (001) diffraction peak at $10.46^{\circ}$ illustrates that most of the natural graphite has been oxidized into GO. All the diffraction peaks of the pure $\mathrm{WO}_{3}$ nanowire clusters can be indexed to hexagonal $\mathrm{WO}_{3}$ (JCPDS card no. 33-1387). There are no peaks detected for other phases, indicating that single phase of $\mathrm{WO}_{3}$ with high purity has been synthesized. The main diffraction peaks of the $\mathrm{GE}-\mathrm{WO}_{3}$ nanowire clusters with $2.0 \mathrm{wt} \% \mathrm{GE}$ are similar to that of pure $\mathrm{WO}_{3}$ nanowire clusters; no diffraction peak of GO can be seen, indicating the great of $\mathrm{GO}$ has been reduced to $\mathrm{GE}$ on this occasion. However, no characteristic peaks of GE are presented in the $\mathrm{WO}_{3} @ \mathrm{GE}$ nanocomposites because of the small content of GE used [28].

Raman spectra of the $\mathrm{GO}$ and $\mathrm{GE}-\mathrm{WO}_{3}$ nanowire clusters are shown in Figure 2. The Raman spectrum of GO displays two prominent peaks at around $1595 \mathrm{~cm}^{-1}$ and $1346 \mathrm{~cm}^{-1}$, which correspond to the well-documented $\mathrm{G}$ and $\mathrm{D}$ bands. The $\mathrm{G}$ band corresponds to the $\mathrm{E}_{2 \mathrm{~g}}$ mode observed for $\mathrm{sp}^{2}$ carbon domains, whereas the $\mathrm{D}$ band is associated with $\mathrm{sp}^{3}$ hybridized carbon or structural defects. The Raman spectrum of $\mathrm{GE}$ in $\mathrm{GE}^{-\mathrm{WO}_{3}}$ nanowire clusters also contains both $\mathrm{G}$ and $\mathrm{D}$ bands (at around $1601 \mathrm{~cm}^{-1}$ and $1359 \mathrm{~cm}^{-1}$, resp.). However, an increased D/G intensity ratio is also observed in comparison with that of the GO spectrum. This change suggests a decrease in the average size of the $\mathrm{sp}^{2}$ domains 


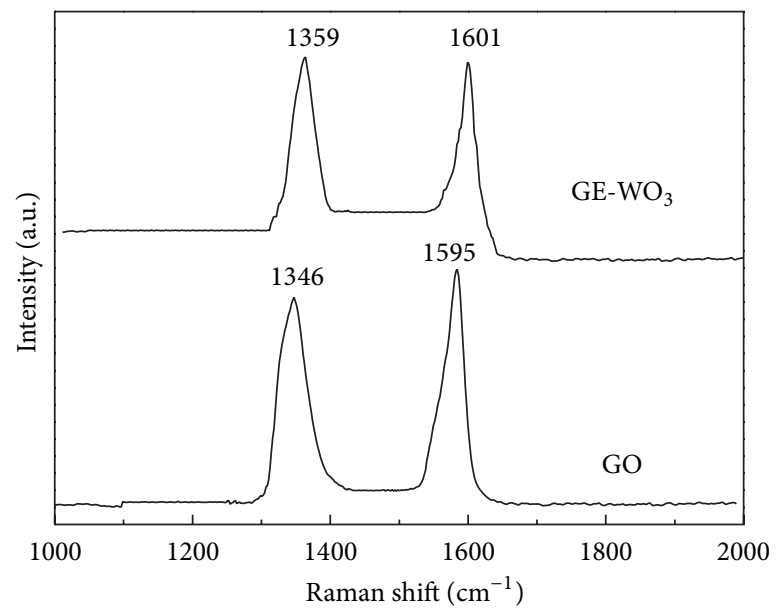

FIGURE 2: Raman spectra of GO and GE-WO $\mathrm{WO}_{3}$ nanowire clusters.

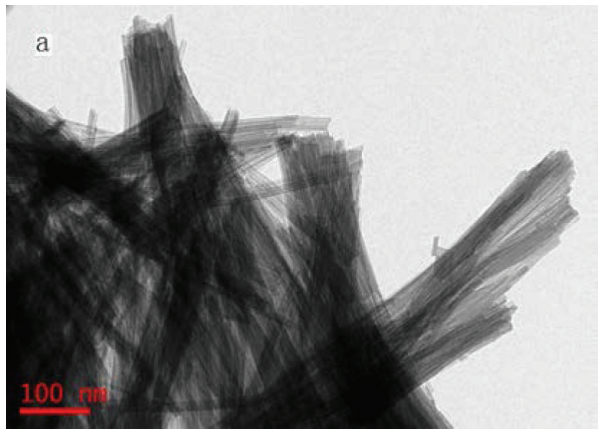

(a)

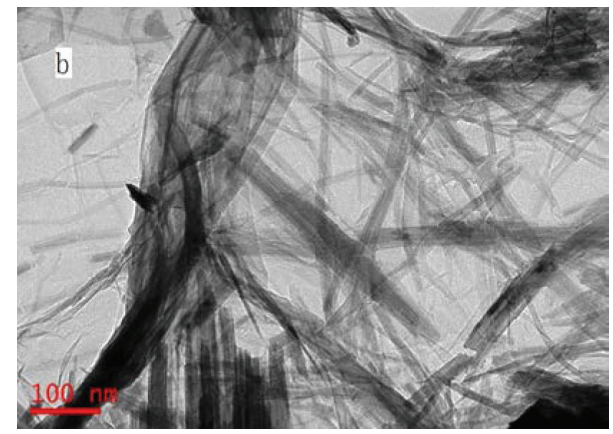

(b)

FIGURE 3: TEM images of (a) $\mathrm{WO}_{3}$ nanowire clusters and (b) GE-WO $\mathrm{WO}_{3}$ nanowire clusters.

upon reduction of the exfoliated GO and can be explained by the creation of numerous new graphitic domains that are smaller in size than the ones presented in exfoliated GO.

Figure 3 shows the TEM images of $\mathrm{WO}_{3}$ nanowire clusters and $\mathrm{GE}^{-\mathrm{WO}_{3}}$ nanowire clusters with $2.0 \mathrm{wt} \% \mathrm{GE}$. It is apparent that the $\mathrm{WO}_{3}$ samples display nanowire cluster-like morphology. Careful examination of the TEM image reveals that many thinner nanowires assembled together along the axis direction form nanowire clusters. The morphology of the GE- $\mathrm{WO}_{3}$ nanowire clusters is consisting of $\mathrm{WO}_{3}$ nanowire clusters and thin stacked flakes. It is obvious that $\mathrm{WO}_{3}$ nanowire clusters are uniformly dispersed within the GE nanosheets. This nanostructure enables a multichannel environment to facilitate the efficient charge interaction.

$\mathrm{X}$-ray photoelectron spectroscopy (XPS) is an effective technique to analyze surface chemical states. The C1s XPS spectra of $\mathrm{GO}$ and the $\mathrm{GE}-\mathrm{WO}_{3}$ nanowire clusters with $2.0 \mathrm{wt} \% \mathrm{GE}$ are shown in Figure 4, respectively. For the GO, the peak with a binding energy of $284.6 \mathrm{eV}$ can be attributed to the $\mathrm{C}-\mathrm{C}$ and $\mathrm{C}-\mathrm{H}$ bonds, while the other three peaks centered at the binding energies of 286.3, 287.7, and $289.2 \mathrm{eV}$ can be assigned to the $\mathrm{C}-\mathrm{O}, \mathrm{C}=\mathrm{O}$, and $\mathrm{O}=\mathrm{C}-\mathrm{OH}$ functional groups, respectively. For the $\mathrm{GE}-\mathrm{WO}_{3}$ nanowire clusters, the relative intensity of the $\mathrm{sp}^{2}$ carbon $(\mathrm{C}-\mathrm{C}, 284.6 \mathrm{eV})$ shows a significant increase, suggesting a sufficient reduction of GO to GE.

Figure 5 shows the FT-IR spectra of the GO and $\mathrm{GE}-\mathrm{WO}_{3}$ nanowire clusters with $2.0 \mathrm{wt} \% \mathrm{GE}$. For the GO, the broad absorption band at $3430.11 \mathrm{~cm}^{-1}$ is related to the stretching peak of the $\mathrm{C}-\mathrm{OH}$ group, and the characteristic absorption bands of GO are observed at $984.21 \mathrm{~cm}^{-1}$ (epoxy stretching), $1099.91 \mathrm{~cm}^{-1}$ (alkoxy C-O stretching), $1224.89 \mathrm{~cm}^{-1}$ (phenolic C-OH stretching), $1401.85 \mathrm{~cm}^{-1}$ (carboxyl O-H stretching), and $1723.76 \mathrm{~cm}^{-1}$ ( $\mathrm{C}=\mathrm{O}$ stretching vibrations of carboxyl or carbonyl groups). The peak at $1624.76 \mathrm{~cm}^{-1}$ is related to $\mathrm{H}-\mathrm{O}-\mathrm{H}$ bending band of the adsorbed $\mathrm{H}_{2} \mathrm{O}$ molecules or the in-plane vibrations of $\mathrm{sp}^{2}$ hybridized $\mathrm{C}-\mathrm{C}$ bonding. For the $\mathrm{GE}-\mathrm{WO}_{3}$ nanowire clusters, as compared to the peaks of the functional groups of GO, the broad absorption peak at $866.71 \mathrm{~cm}^{-1}$ is ascribed to the vibration of $\mathrm{W}-\mathrm{O}-\mathrm{W}$ bond. It is also found that the peaks at $1723.76 \mathrm{~cm}^{-1}, 1224.89 \mathrm{~cm}^{-1}$, and $1089.91 \mathrm{~cm}^{-1}$ in the composites have disappeared, suggesting a sufficient reduction of $\mathrm{GO}$ to GE.

The UV-vis diffuse reflectance spectra of the $\mathrm{WO}_{3}$

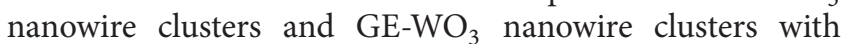
$2.0 \mathrm{wt} \% \mathrm{GE}$ are shown in Figure 6. According to the spectrum, the $\mathrm{WO}_{3}$ nanowire clusters show a sharp edge at about 


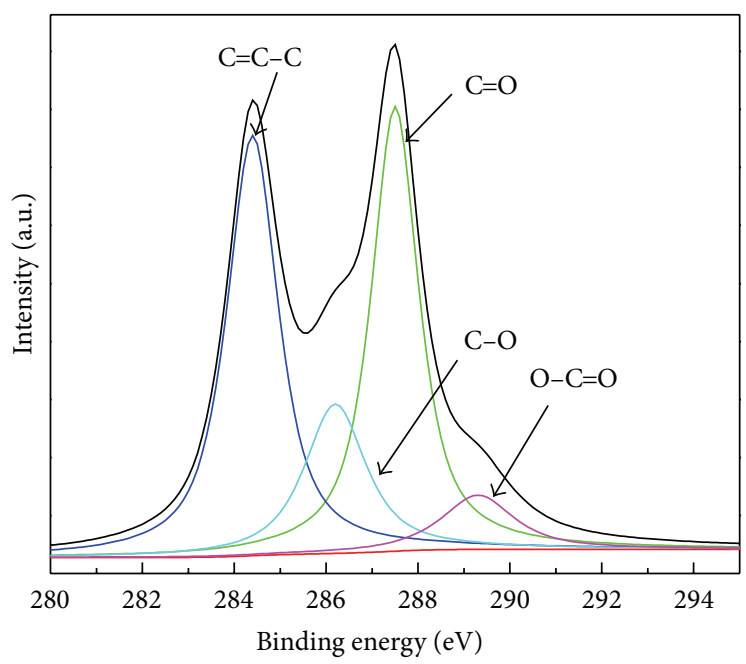

(a)

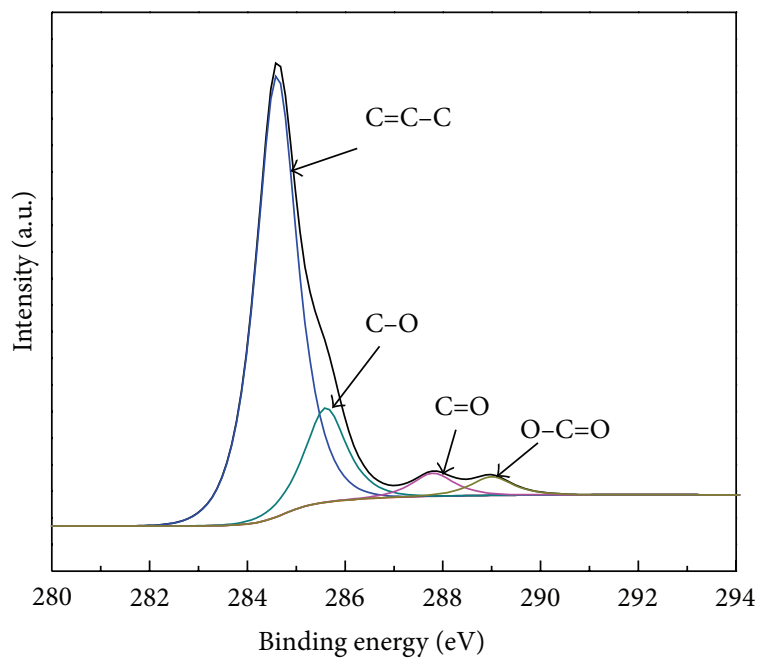

(b)

FIGURE 4: The Cls XPS spectra of (a) GO and (b) GE-WO $\mathrm{WO}_{3}$ nanowire clusters.

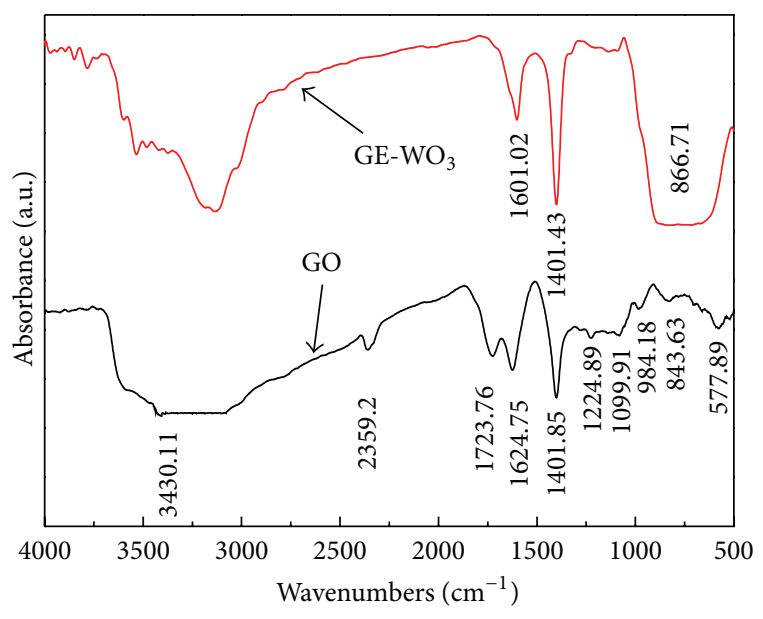

FIGURE 5: FT-IR spectra of GO and GE-WO $\mathrm{W}_{3}$ nanowire clusters.

$\sim 465 \mathrm{~nm}$, whereas the GE- $\mathrm{WO}_{3}$ nanowire clusters display an obvious red shift in the absorption edge. This result indicates that the $\mathrm{GE}-\mathrm{WO}_{3}$ nanowire clusters can be excited in visible region because of the existence of $\mathrm{W}-\mathrm{O}-\mathrm{C}$ bond, similar to those reported in hybridization of $\mathrm{Bi}_{2} \mathrm{WO}_{6}$ with graphitelike carbon layers [14]. Therefore, we can infer that the introduction of $\mathrm{GE}$ in $\mathrm{WO}_{3}$ nanowire clusters is effective for the visible light response of the photocatalyst.

The photocatalytic activities of the as-prepared GE- $\mathrm{WO}_{3}$ nanowire clusters with different weight ratios of GE in terms of evolved $\mathrm{O}_{2}$ from water splitting are measured, and the results are shown in Figure 7. For bare $\mathrm{WO}_{3}$ nanowire clusters, the amount of evolved $\mathrm{O}_{2}$ from water splitting after $8 \mathrm{~h}$ is $c a .0 .175 \mathrm{mmol} / \mathrm{L}$. The photocatalytic activity of the GE- $\mathrm{WO}_{3}$ nanowire clusters can be enhanced with increasing the weight ratio of GO up to $2.0 \mathrm{wt} \%$. This result indicates that the $\mathrm{GE}-\mathrm{WO}_{3}$ nanowire clusters with $2.0 \mathrm{wt} \%$ $\mathrm{GE}$ exhibit the maximum evolved $\mathrm{O}_{2}$ from water splitting

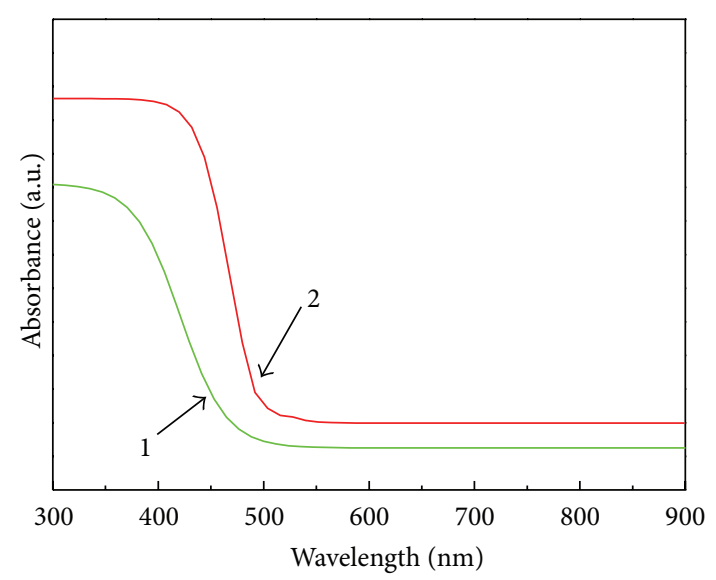

(1) $\mathrm{WO}_{3}$

(2) $\mathrm{GE}-\mathrm{WO}_{3}$

FIGURE 6: UV-vis diffuse reflectance spectra of $\mathrm{WO}_{3}$ nanowire clusters and $\mathrm{GE}-\mathrm{WO}_{3}$ nanowire clusters.

(ca. $0.345 \mathrm{mmol} / \mathrm{L}$ ). This is attributed to GE which serves as an acceptor of the electrons generated in the $\mathrm{WO}_{3}$ and effectively decreases the recombination probability of the photogenerated electron-hole pairs. Further increasing the weight ratio of $\mathrm{GO}$ will lead to a decrease, especially in GE$\mathrm{WO}_{3}$ nanowire clusters with $4.0 \mathrm{wt} \%$ GE. It is reasonable because the introduction of a large percentage of black GE leads to shielding of the active sites on the catalyst surface [19].

The efficient charge separation and transfer are crucial for the enhanced photocatalytic activity of the GE$\mathrm{WO}_{3}$ nanowire clusters. GE has a charge-carrier mobility of $200000 \mathrm{~cm}^{2} \mathrm{~V}^{-1} \mathrm{~s}^{-1}$ at room temperature, so it is very possible that the incorporation of GE may enhance the charge separation efficiency and suppress the charge recombination as suggested in Figure 8. Under visible light irradiation, electrons $\left(\mathrm{e}^{-}\right)$and holes $\left(\mathrm{h}^{+}\right)$are generated in the $\mathrm{WO}_{3}$ nanowire 


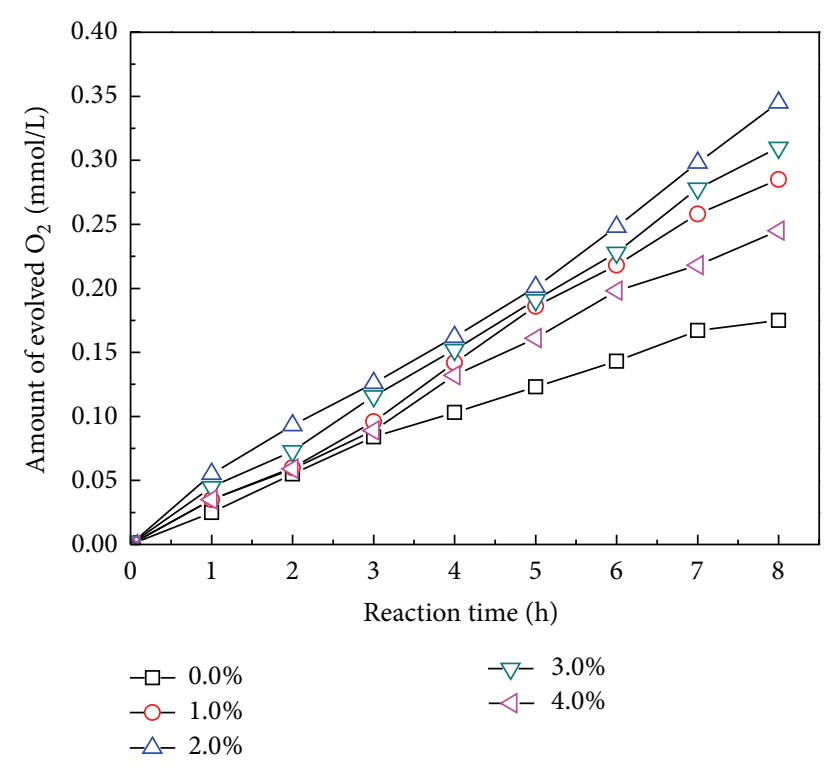

FIGURE 7: Amount of evolved $\mathrm{O}_{2}$ for the different $\mathrm{GE}-\mathrm{WO}_{3}$ nanowire clusters.

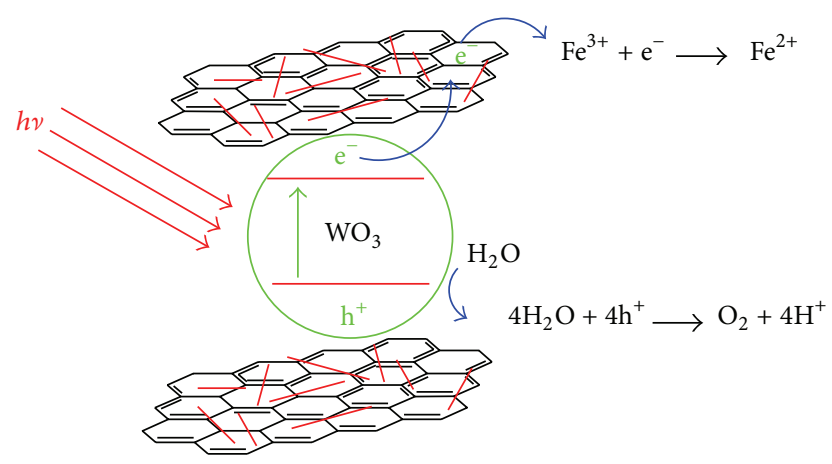

Figure 8: The possible photocatalytic mechanism of the GE-WO nanowire clusters.

clusters (see (1)). $\mathrm{WO}_{3}$ is photocatalyst with weak reducing power, which prohibits the electron transfer to reduce $\mathrm{H}_{2} \mathrm{O}$ to $\mathrm{H}_{2}$. Therefore, only holes reaction with water to produce $\mathrm{O}_{2}$ can occur spontaneously by using the photocatalyst (see (2)). However, photogenerated electrons can transfer to carbon atoms on the graphene sheets and then react with $\mathrm{Fe}^{3+}$ ions as the scavenger to reduce the recombination of electron-hole pairs (see (3)). Thus, GE serves as the photogenerated electrons acceptor and effectively suppresses the charge recombination in the $\mathrm{GE}-\mathrm{WO}_{3}$ nanowire clusters, leaving more positive charged holes on the $\mathrm{WO}_{3}$ surface and promoting the production of oxygen:

$$
\begin{gathered}
\mathrm{WO}_{3} \stackrel{h v}{\longrightarrow} \mathrm{e}^{-}+\mathrm{h}^{+} \\
2 \mathrm{H}_{2} \mathrm{O}+4 \mathrm{~h}^{+} \longrightarrow 4 \mathrm{H}^{+}+\mathrm{O}_{2} \\
\mathrm{Fe}^{3+}+\mathrm{e}^{-} \longrightarrow \mathrm{Fe}^{2+}
\end{gathered}
$$

\section{Conclusions}

In summary, $\mathrm{GE}-\mathrm{WO}_{3}$ nanowire clusters were synthesized by a facile hydrothermal method. The GE- $\mathrm{WO}_{3}$ nanowire clusters with $2.0 \mathrm{wt} \%$ GE exhibited enhanced performance compared to pure $\mathrm{WO}_{3}$ nanowire clusters for $\mathrm{O}_{2}$ evolution from water splitting. The sensitization of $\mathrm{WO}_{3}$ nanowire clusters by $\mathrm{GE}$ enhanced the visible light absorption property of $\mathrm{GE}-\mathrm{WO}_{3}$ nanowire clusters. The chemical bonding between $\mathrm{WO}_{3}$ and GE reduced the recombination of the photogenerated electron-hole pairs, leading to improved photocatalytic activity.

\section{Conflict of Interests}

The authors declare that there is no conflict of interests regarding the publication of this paper.

\section{Acknowledgments}

This work was supported by the Natural Science Foundation of China (Grants nos. 51272075, 51372080) and the China Postdoctoral Science Foundation (2012M521220). This work was also financially supported by the Department of Education of Hunan Province, China (Grant no. 11B054).

\section{References}

[1] A. Fujishima and K. Honda, " $\mathrm{TiO}_{2}$ photoelectrochemistry and photocatalysis," Nature, vol. 238, no. 5358, pp. 37-38, 1972.

[2] S. Sun, W. Wang, S. Zeng, M. Shang, and L. Zhang, "Preparation of ordered mesoporous $\mathrm{Ag} / \mathrm{WO}_{3}$ and its highly efficient degradation of acetaldehyde under visible-light irradiation," Journal of Hazardous Materials, vol. 178, no. 1-3, pp. 427-433, 2010.

[3] J. Kim, C. W. Lee, and W. Choi, "Platinized $\mathrm{WO}_{3}$ as an environmental photocatalyst that generates $\mathrm{OH}$ radicals under visible light," Environmental Science \& Technology, vol. 44, no. 17, pp. 6849-6854, 2010.

[4] U. A. Joshi, J. R. Darwent, H. H. P. Yiu, and M. J. Rosseinsky, "The effect of platinum on the performance of $\mathrm{WO}_{3}$ nanocrystal photocatalysts for the oxidation of Methyl Orange and isopropanol," Journal of Chemical Technology and Biotechnology, vol. 86, no. 8, pp. 1018-1023, 2011.

[5] Y. P. Xie, G. Liu, L. C. Yin, and H. M. Cheng, "Crystal facetdependent photocatalytic oxidation and reduction reactivity of monoclinic $\mathrm{WO}_{3}$ for solar energy conversion," Journal of Materials Chemistry, vol. 22, no. 14, pp. 6746-6751, 2012.

[6] J. Guo, Y. Li, S. Zhu et al., "Synthesis of $\mathrm{WO}_{3} @ g r a p h e n e$ composite for enhanced photocatalytic oxygen evolution from water," RSC Advances, vol. 2, no. 4, pp. 1356-1363, 2012.

[7] P. Cheng, Z. Yang, H. Wang et al., " $\mathrm{TiO}_{2}$-graphene nanocomposites for photocatalytic hydrogen production from splitting water," International Journal of Hydrogen Energy, vol. 37, no. 3, pp. 2224-2230, 2012.

[8] H. Zheng and M. Mathe, "Hydrogen evolution reaction on single crystal $\mathrm{WO}_{3} / \mathrm{C}$ nanoparticles supported on carbon in acid and alkaline solution," International Journal of Hydrogen Energy, vol. 36, no. 3, pp. 1960-1964, 2011. 
[9] X. An, J. C. Yu, Y. Wang, Y. Hu, X. Yu, and G. Zhang, "WO nanorods/graphene nanocomposites for high-efficiency visiblelight-driven photocatalysis and $\mathrm{NO}_{2}$ gas sensing," Journal of Materials Chemistry, vol. 22, no. 17, pp. 8525-8531, 2012.

[10] Y. H. Ng, A. Iwase, A. Kudo, and R. Amal, "Reducing graphene oxide on a visible-light $\mathrm{BiVO}_{4}$ photocatalyst for an enhanced photoelectrochemical water splitting," Journal of Physical Chemistry Letters, vol. 1, no. 17, pp. 2607-2612, 2010.

[11] J. Liu, H. Bai, Y. Wang, Z. Liu, X. Zhang, and D. D. Sun, "Selfassembling $\mathrm{TiO}_{2}$ nanorods on large graphene oxide sheets at a two-phase interface and their anti-recombination in photocatalytic applications," Advanced Functional Materials, vol. 20, no. 23, pp. 4175-4181, 2010.

[12] F. Wang and K. Zhang, "Reduced graphene oxide- $\mathrm{TiO}_{2}$ nanocomposite with high photocatalystic activity for the degradation of rhodamine B," Journal of Molecular Catalysis A: Chemical, vol. 345, no. 1-2, pp. 101-107, 2011.

[13] Y. Zhang and C. Pan, " $\mathrm{TiO}_{2} /$ graphene composite from thermal reaction of graphene oxide and its photocatalytic activity in visible light," Journal of Materials Science, vol. 46, no. 8, pp. 2622-2626, 2011.

[14] E. Gao, W. Z. Wang, M. Shang, and J. H. Xu, "Synthesis and enhanced photocatalytic performance of graphene- $\mathrm{Bi}_{2} \mathrm{WO}_{6}$ composite," Physical Chemistry Chemical Physics, vol. 13, no. 7, pp. 2887-2893, 2011.

[15] Y. Y. Wen, H. M. Ding, and Y. K. Shan, "Preparation and visible light photocatalytic activity of $\mathrm{Ag} / \mathrm{TiO}_{2}$ /graphene nanocomposite," Nanoscale, vol. 3, no. 10, pp. 4411-4417, 2011.

[16] H. Zhang, X. Fan, X. Quan, S. Chen, and H. Yu, "Graphene sheets grafted Ag@AgCl hybrid with enhanced plasmonic photocatalytic activity under visible light," Environmental Science and Technology, vol. 45, no. 13, pp. 5731-5736, 2011.

[17] P. Wang, L. Han, C. z. Zhu, Y. M. Zhai, and S. J. Dong, "Aqueousphase synthesis of $\mathrm{Ag}-\mathrm{TiO}_{2}$-reduced graphene oxide and Pt$\mathrm{TiO}_{2}$-reduced graphene oxide hybrid nanostructures and their catalytic properties," Nano Research, vol. 4, no. 11, pp. 1153-1162, 2011.

[18] Y. Wang, J. Liu, L. Liu, and D. D. Sun, "High-quality reduced graphene oxide-nanocrystalline platinum hybrid materials prepared by simultaneous co-reduction of graphene oxide and chloroplatinic acid," Nanoscale Research Letters, vol. 6, pp. 241249, 2011.

[19] Q. Li, B. Guo, J. Yu et al., "Highly efficient visible-light-driven photocatalytic hydrogen production of CdS-cluster-decorated graphene nanosheets," Journal of the American Chemical Society, vol. 133, no. 28, pp. 10878-10884, 2011.

[20] J. W. Jang, S. Cho, G. Moon et al., "Photocatalytic synthesis of pure and water-dispersible graphene monosheets," Chemistry: A European Journal, vol. 18, no. 10, pp. 2762-2767, 2012.

[21] Q. J. Xiang, J. G. Yu, and M. Jaroniec, "Graphene-based semiconductor photocatalysts," Chemical Society Reviews, vol. 41, no. 2, pp. 782-796, 2012.

[22] D. G. Yin, L. Zhang, B. H. Liu, and M. H. Wu, "Preparation and characterization of ZnO-graphene composite photocatalyst," Journal of Nanoscience and Nanotechnology, vol. 12, no. 2, pp. 937-942, 2012.

[23] Q. Xiang, J. Yu, and M. Jaroniec, "Synergetic effect of $\mathrm{MoS}_{2}$ and graphene as cocatalysts for enhanced photocatalytic $\mathrm{H}_{2}$ production activity of $\mathrm{TiO}_{2}$ nanoparticles," Journal of the American Chemical Society, vol. 134, no. 15, pp. 6575-6578, 2012.

[24] J. G. Hou, Z. Wang, W. B. Kan, S. Q. Jiao, H. M. Zhu, and R. V. Kumar, "Efficient visible-light-driven photocatalytic hydrogen production using CdS@TaON core-shell composites coupled with graphene oxide nanosheets," Journal of Materials Chemistry, vol. 22, no. 15, pp. 7291-7299, 2012.

[25] J. Guo, Y. Li, S. Zhu et al., "Synthesis of $\mathrm{WO}_{3} @$ Graphene composite for enhanced photocatalytic oxygen evolution from water," RSC Advances, vol. 2, no. 4, pp. 1356-1363, 2012.

[26] Y. H. Ng, A. Iwase, N. J. Bell, A. Kudo, and R. Amal, "Semiconductor/reduced graphene oxide nanocomposites derived from photocatalytic reactions," Catalysis Today, vol. 164, no. 1, pp. 353-357, 2011.

[27] H. Pang, X. Xiang, Z. Li, Y. Fu, and X. Zu, "Hydrothermal synthesis and optical properties of hexagonal tungsten oxide nanocrystals assisted by ammonium tartrate," Physica Status Solidi (A) Applications and Materials Science, vol. 209, no. 3, pp. 537-544, 2012.

[28] H. Ying, Z. Wang, Z. Guo, Z. Shi, and S. Yang, "Reduced graphene oxide-modified $\mathrm{Bi}_{2} \mathrm{WO}_{6}$ as an improved photocatalyst under visible light," Acta Physico-Chimica Sinica, vol. 27, no. 6, pp. 1482-1486, 2011. 

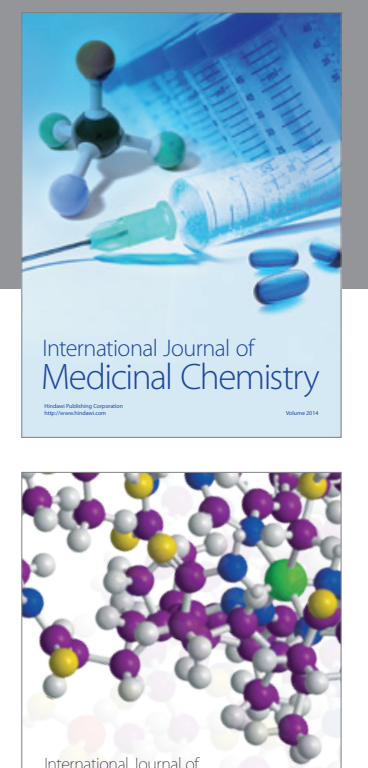

\section{Carbohydrate} Chemistry

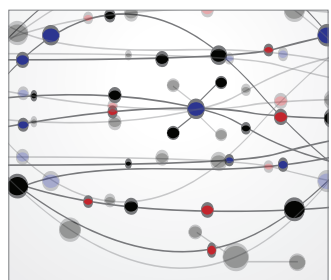

The Scientific World Journal
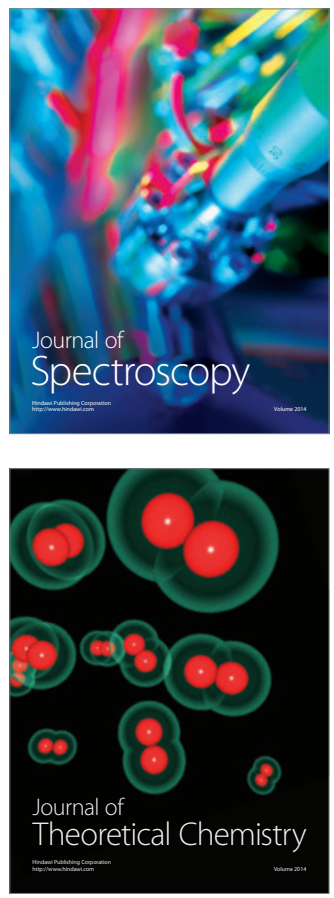
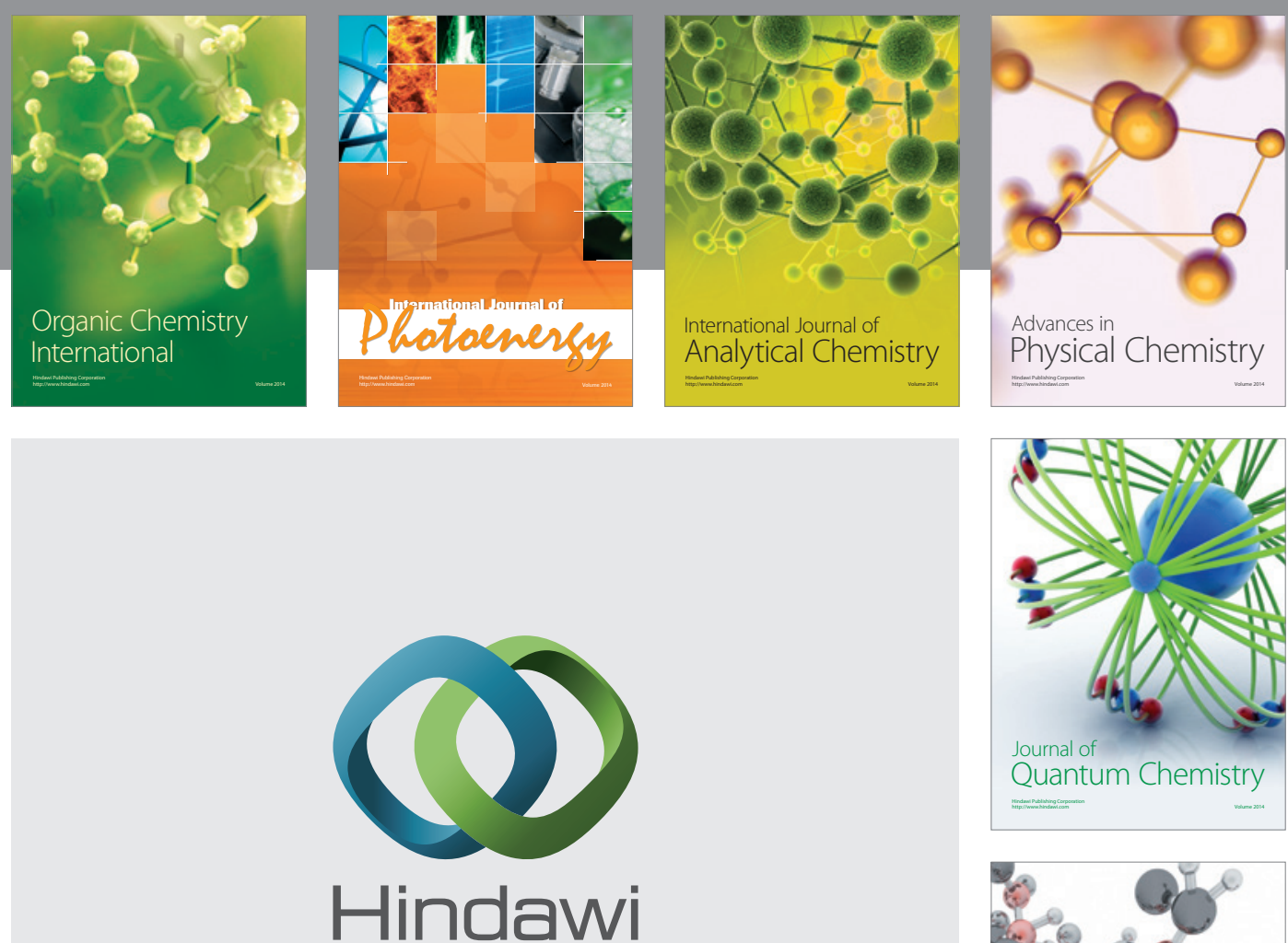

Submit your manuscripts at

http://www.hindawi.com

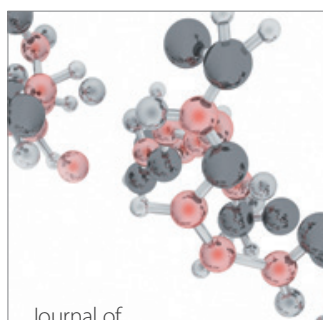

Analytical Methods

in Chemistry

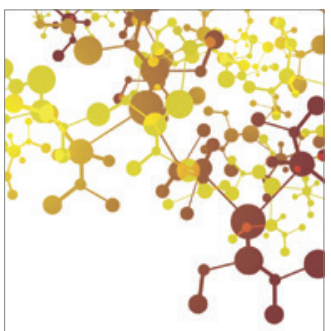

Journal of

Applied Chemistry

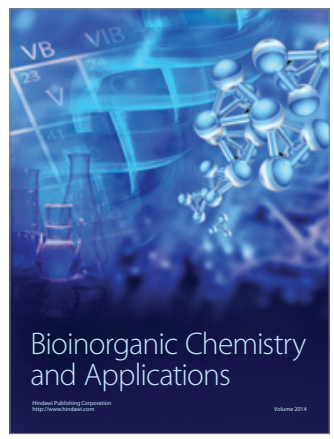

Inorganic Chemistry
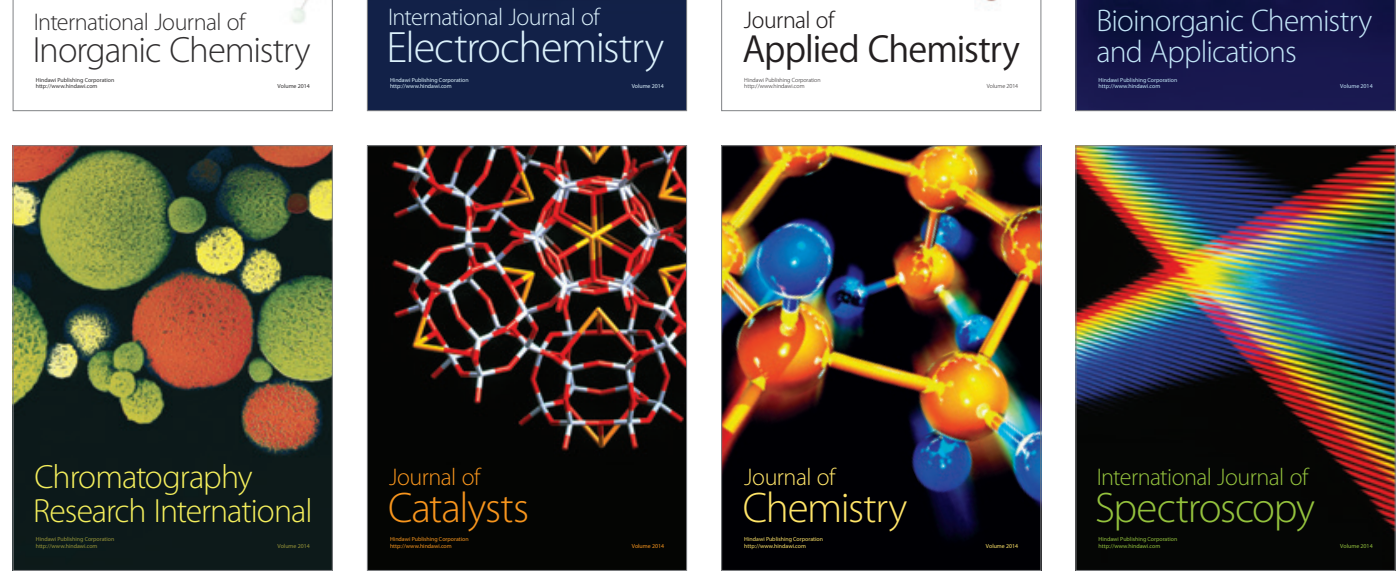